(c) American Dairy Science Association, 2005.

\title{
Application of a Finite Mixture Model to Somatic Cell Scores of Italian Goats
}

\author{
P. J. Boettcher, ${ }^{1}$ P. Moroni, ${ }^{2}$ G. Pisoni, ${ }^{2}$ and D. Gianola ${ }^{3}$ \\ ${ }^{1}$ Institute of Biology and Biotechnology of Agriculture, \\ National Research Council, 20090 Segrate, Italy \\ ${ }^{2}$ Department of Animal Pathology, Hygiene and Veterinary Public Health, \\ University of Milano, 20133 Milano, Italy \\ ${ }^{3}$ Department of Animal Sciences, University of Wisconsin, Madison 53706
}

\begin{abstract}
The objectives of this study were to apply a finite mixture model (FMM) to data for somatic cell count in goats and to compare the fit of the FMM with that of a standard linear mixed effects model. Bacteriological information was used to assess the ability of the model to classify records from healthy or infected goats. Data were 4518 observations of somatic cell score (SCS) and bacterial infection from both udder halves of 310 goats from 5 herds in Northern Italy. The records were from a complete production season, and were taken monthly from February to November 2000. Explanatory factors in both models included a 3-parameter regression on days in milk (DIM); fixed class effects of herd-test-day, parity group, and udder side (left or right); and random effects of goat and udder half within goat. In addition, the 2-component FMM included a fixed mean for the second component of the model (theoretically corresponding to infected udder halves), as well as an unknown probability of membership to a given putative infection status. A Bayesian statistical approach was used for the analysis with Gibbs sampling used to obtain draws from posterior distributions of parameters of interest. Two sampling chains of 200,000 cycles each were generated for each model. The FMM yielded a much lower estimate of residual variance than the standard model (1.28 vs. $\left.3.02 \mathrm{SCS}^{2}\right)$, and a slightly higher estimate for the between-goat variance (1.79 vs. 1.48). The deviance information criterion (DIC) was used to compare the fit of the 2 models. The DIC was much lower for the FMM, indicating a better fit to the data. The FMM was able to classify correctly 60 and $48 \%$ of the healthy and infected observations, respectively. This was slightly higher than what would be expected from random classification, but not high enough for useful mastitis diagnosis. Nevertheless, increased pre-
\end{abstract}

Received November 9, 2004.

Accepted December 19, 2004.

Corresponding author:Paul Boettcher; e-mail: boettch@ibba.cnr.it. cision of genetic evaluation is the goal of applying the FMM, rather than timely and accurate mastitis diagnosis. The results suggest that more research on FMM for SCS is merited and necessary for proper application. (Key words: goat, mastitis, somatic cell count, finite mixture model)

Abbreviation key: DIC = deviance information criterion, $\mathbf{F M M}=$ finite mixture model, $\mathbf{P M C}=$ proportion of misclassification, SLM = standard linear mixed effects model.

\section{INTRODUCTION}

Even though mastitis is the most common and most costly disease for dairy production, many countries do not routinely record cases of mastitis in a systematic manner. Hence, direct selective breeding for resistance to infection is not feasible. Rather, selection programs to decrease mastitis infection in dairy cattle are often based on SCC or SCS (Interbull, 1996). Genetic evaluation models often treat SCS as a different trait in different lactations, or use random regression to account for animal-specific trajectories of SCS within a lactation. However, no distinction is made between SCS data from infected and uninfected animals (at least partially due to the lack of such information). Detilleux and Leroy (2000), however, suggested that SCS may differ depending on infection status, and proposed the use of a finite mixture model (FMM) to help account for these differences. An FMM can represent a latent structure in a set of data whereby observations may belong to one of several distributions, possibly differing in mean, variance, and even the type of distribution (McLachlan and Peel, 2000). The FMM has been previously used in different applications to animal breeding, including QTL mapping, e.g., Liu et al. (2004).

Recent work has been done to extend the ideas of Detilleux and Leroy (2000) for development of an FMM for SCS. Gianola et al. (2004) refined the models of Detilleux and Leroy (2000), and proposed algorithms for parameter estimation using maximum likelihood. 
Ødegard et al. (2003) outlined a Bayesian approach for solving FMM, and applied it to simulated data for SCS. In their simulations, Ødegard et al. (2003) evaluated the ability of their procedure to estimate various parameters of the model (such as variances of different effects) and the quality of classification of individual records as belonging to one of the 2 components of the FMM. The objectives of our study were to apply an approach similar to that of Ødegard et al. (2003) to real data for SCS, and to compare the fit of the FMM to the data with that of a single-component, mixed effects, standard linear model (SLM). Data from goats were used in this study, which presented particular challenges to the FMM, inasmuch as SCS are considered less reliable indicators of mastitis in cattle than in goats (e.g., Poutrel and Lerondelle, 1983; Droke et al., 1993; Aleandri et al., 1996). As an additional test of the FMM, data for bacterial infection were used to evaluate the ability of the FMM to correctly classify observations as being from healthy or infected udder halves.

\section{MATERIALS AND METHODS}

\section{Data}

The study was based on records of SCC and bacterial infection status in milk from 310 goats in 5 Italian herds for a complete production season (January to November 2000). A detailed description of the herds and data collection procedures (including culturing of bacteria) is given in Moroni et al. (accepted). Each herd was visited monthly from February to November (i.e., 10 visits per herd). At each visit, a separate sample of milk was taken from each udder half from each goat. The SCC was recorded for each udder half, and milk from each half was cultured for identification of any bacteria present. Clinical mastitis is generally uncommon in goats (Bergonier et al., 2003) and no clinical signs of mastitis were observed in the goats sampled for this study. Thus, all infections observed could be considered subclinical.

The resulting data set included 4518 test-day records, with an average of 7.3 test-days per goat. The SCC were converted to SCS using the standard $\log 2$ transformation of Ali and Shook (1980). Bacterial infections could be assigned to 1 of 5 categories: 1) healthy (no bacteria present), 2) infected with Staphylococcus aureus, 3) infected by a CNS species, 4) infected by Streptococcus species, and 5) infected by another bacterial species. In general, bacteria falling into the final category were environmental pathogens.

\section{Statistical Analyses}

Data for SCS were analyzed using 2 models. The first model was an SLM, where all observations were assumed to follow a normal distribution, with appropriate covariates included to represent heterogeneity in the population. The second model was an FMM with 2 components. Here, each observation was assumed to be drawn from 1 of 2 distinct distributions differing according to their mean, but without knowing which of the 2 processes generated the draw. The distribution of observations from the first component was restricted to have a lower mean, and was interpreted to be data representing uninfected udder halves. The distribution of observations in the second component of the FMM had a higher mean, and was assumed to represent records from infected udder halves. Using notation similar to that of Ødegard et al. (2003), both models can be described in matrix form by the following equation:

$$
\mathbf{y}=\mathbf{X}_{\mathbf{0}} \boldsymbol{\beta}_{\mathbf{0}}+\mathbf{M}_{\mathbf{z}} \mathbf{X}_{\mathbf{1}} \boldsymbol{\beta}_{\mathbf{1}}+\mathbf{Z}_{\mathrm{g}} \mathbf{g}+\mathbf{Z}_{\mathbf{h}} \mathbf{h}+\mathbf{e}
$$

where $\mathbf{y}$ is a vector of observations of test-day SCS; $\boldsymbol{\beta}_{\mathbf{0}}$ is a vector of fixed effects common to all udder halves, regardless of infection status; $\boldsymbol{\beta}_{\boldsymbol{1}}$ is a vector of fixed effects common to infected udder halves; $\mathbf{M}_{\mathbf{z}}$ is a diagonal matrix of indicator variables with elements $\mathrm{M}(\mathrm{i}, \mathrm{i})=$ 0 for records corresponding to the first component (healthy, with prior probability $1-\mathrm{P}_{\mathrm{m}}$, where $\mathrm{P}_{\mathrm{m}}$ is the mixing proportion or unknown proportion of putatively infected records) or $\mathrm{M}(\mathrm{i}, \mathrm{i})=1$ for records from the second component (infected), with prior probability $\mathrm{P}_{\mathrm{m}} ; \mathbf{g}$ is a vector of random goat effects for all observations; $\mathbf{h}$ is a vector of random udder-half effects; $\mathbf{X}_{\mathbf{0}}, \mathbf{X}_{\mathbf{1}}, \mathbf{Z}_{\mathrm{g}}$, and $\mathbf{Z}_{\mathbf{h}}$ are incidence matrices of appropriate order, and $\mathbf{e}$ is a vector of random residuals. Goat effects were assumed to be uncorrelated, as genetic relationships were not considered in either model because pedigree information was not available for approximately $50 \%$ of the animals in the study, and only a small fraction of recorded sires were used in more than one herd.

Elements in the $\boldsymbol{\beta}_{\mathbf{0}}$ vector included 3 regression coefficients for the effect of DIM on SCS, and class effects of herd-test-day (50 levels), parity group, and udder side (left or right). The regression coefficients for DIM were those of Wilmink's (1987) curve, i.e., an intercept and regressions on DIM, and on $\exp ^{(-0.05}$ DIM $)$ Parity groups consisted of separate classes for first through fifth lactations and a pooled class for all animals with 6 or more lactations. The $\boldsymbol{\beta}_{1}$ vector included a single element, which could be interpreted as the difference in mean values of the 2 components (i.e., between infected and healthy udder halves). The SLM is obtained by taking $\mathbf{M}_{\mathbf{z}}$ in [1] to be a null matrix.

All variances were assumed to be homogeneous for both the SLM and the FMM. The conditional distribution of $\mathbf{y}$, given the fixed and random effects, was assumed to be: 


$$
\begin{gathered}
\mathbf{y} \mid \beta_{\mathbf{0}}, \boldsymbol{\beta}_{\mathbf{1}}, \mathbf{g}, \mathbf{h}, \boldsymbol{\sigma}_{\mathrm{g}}^{2}, \boldsymbol{\sigma}_{\mathrm{h}}^{2}, \boldsymbol{\sigma}_{\mathrm{e}}^{2}, \mathbf{z} \\
\sim \mathrm{N}\left(\mathbf{X}_{\mathbf{0}} \boldsymbol{\beta}_{\mathbf{0}}+\mathbf{M}_{\mathbf{z}} \mathbf{X}_{\mathbf{1}} \boldsymbol{\beta}_{\mathbf{1}}+\mathbf{Z}_{\mathbf{g}} \mathbf{g}+\mathbf{Z}_{\mathbf{h}} \mathbf{h}, \mathbf{I} \boldsymbol{\alpha}_{\mathrm{e}}^{2}\right),
\end{gathered}
$$

where $\mathbf{z}$ is a vector containing the diagonal elements of $\mathbf{M}_{\mathbf{z}}$ indicating group membership and $\sigma_{\mathrm{g}}^{2}, \sigma_{\mathrm{h}}^{2}, \sigma_{\mathrm{e}}^{2}$ are variances for goat, udder-half, and residual, respectively. The $\mathbf{g}$ and $\mathbf{h}$ vectors had conditional (upon $\sigma_{\mathrm{g}}^{2}$ and $\sigma_{\mathrm{h}}^{2}$ ) normal distributions with null expectations and variance-covariance matrices $\mathbf{I} \sigma_{\mathrm{g}}^{2}$ and $\mathbf{I} \sigma_{\mathrm{h}}^{2}$, respectively.

Posterior distributions of the various parameters were obtained via Gibbs sampling using minor modifications of the Bayesian approach described by Ødegard et al. (2003). Briefly, prior distributions were Bernoulli for all elements of the classification vector $\mathbf{z}$, uniform for fixed effects (bounded by $\pm 100,000$ ), normal distributions for random effects, scale-inverted $\chi^{2}$ for variances, and a Beta distribution for the mixing proportion $\mathrm{P}_{\mathrm{m}}$. All random effects had prior means $=0$. Prior variances were obtained from Maximum Likelihood estimates using the MIXED procedure of SAS (SAS Institute, Inc., Cary, NC) (our unpublished results) and all $\chi^{2}$ distributions had 5 degrees of freedom. The Beta distribution is defined by two parameters, $\alpha_{1}$ and $\alpha_{2}$, and these were assigned a value of 2 each, following the approach of Ødegard et al. (2003).

The Gibbs sampler was then implemented as follows: 1) Fixed effects were sampled piecewise from univariate normal distributions; 2) random effects were sampled from univariate normal distributions; 3) the betweengoat variance was sampled from a scale-inverted $\chi^{2}$ distribution; 4) the variance between udder halves was sampled from a scale inverted $\chi^{2}$ distribution; 5) the residual variance was sampled from a scale-inverted $\chi^{2}$ distribution; 6) group membership variables (i.e., elements of $\mathbf{z}$ ) were sampled from Bernoulli distributions; and 7) the mixing proportion $\mathrm{P}_{\mathrm{m}}$ was sampled from a Beta distribution. In step $1, \boldsymbol{\beta}_{\mathbf{1}}$ was forced to be $>0$, to ensure identifiability and absence of "label switching". In other words, the mean for observations in the second (infected) component was forced to be greater than that for the first component. For group membership (step 6), the Bernoulli distribution differed for each observation and was defined by a parameter $\mathrm{p}_{\mathrm{i}}$, the conditional probability that the record was assigned to the infected group, given $\mathrm{y}_{\mathrm{i}}$ and the values for the other parameters in the model. Specifically,

$$
\begin{aligned}
& \mathrm{p}_{\mathrm{i}}= \\
& \frac{\mathrm{p}\left(\mathrm{y}_{\mathrm{i}} \mid \Theta, \sigma_{\mathrm{e}}^{2}, \mathrm{z}_{\mathrm{i}}=1\right) \mathrm{P}_{\mathrm{m}}}{\mathrm{p}\left(\mathrm{y}_{\mathrm{i}} \mid \Theta, \sigma_{\mathrm{e}}^{2}, \mathrm{z}_{\mathrm{i}}=0\right)\left(1-\mathrm{P}_{\mathrm{m}}\right)+\mathrm{p}\left(\mathrm{y}_{\mathrm{i}} \mid \Theta, \sigma_{\mathrm{e}}^{2}, \mathrm{z}_{\mathrm{i}}=1\right) \mathrm{P}_{\mathrm{m}}} \\
& \text { where } \Theta^{\prime}=\left[\boldsymbol{\beta}_{\mathbf{0}}^{\prime} \boldsymbol{\beta}_{\mathbf{1}}^{\prime} \mathbf{g}^{\prime} \mathbf{h}^{\prime}\right],
\end{aligned}
$$

$$
\begin{gathered}
\mathrm{p}\left(\mathrm{y}_{\mathrm{i}} \mid \Theta, \sigma_{\mathrm{e}}^{2}, \mathrm{z}_{\mathrm{i}}=0\right)= \\
\left(2 \pi \sigma_{\mathrm{e}}^{2}\right)^{-1 / 2} \exp \left[-\frac{1}{2 \sigma_{\mathrm{e}}^{2}}\left(\mathrm{y}_{\mathrm{i}}-\mathbf{x}_{\mathrm{oi}}^{\prime} \boldsymbol{\beta}_{\mathbf{0}}-\mathbf{z}_{\mathrm{gi}}^{\prime} \mathbf{g}-\mathbf{z}_{\mathrm{hi}}^{\prime} \mathrm{h}\right)^{2}\right]
\end{gathered}
$$

and

$$
\begin{gathered}
\mathrm{p}\left(\mathrm{y}_{\mathrm{i}} \mid \Theta, \sigma_{\mathrm{e}}^{2}, \mathrm{z}_{\mathrm{i}}=1\right)= \\
\left(2 \pi \sigma_{\mathrm{e}}^{2}\right)^{-1 / 2} \exp \left[-\frac{1}{2 \sigma_{\mathrm{e}}^{2}}\left(\mathrm{y}_{\mathrm{i}}-\mathbf{x}_{\mathrm{oi}}^{\prime} \boldsymbol{\beta}_{\mathbf{0}}-\mathbf{x}_{\mathrm{oi}}^{\prime} \boldsymbol{\beta}_{\mathbf{1}}-\mathbf{z}_{\mathrm{gi}}^{\prime} \mathbf{g}-\mathbf{z}_{\mathrm{hi}}^{\prime} \mathbf{h}\right)^{2}\right]
\end{gathered}
$$

where the incidence vectors are rows of the appropriate incidence matrices.

Steps 1 to 7 were repeated many times for each chain of the Gibbs sampler. (Only steps 1 to 5 were needed for the single component model.) For this study, 2 chains of 205,000 cycles were generated for each model. The first 5000 rounds from each chain were discarded as a burnin, to ensure sampling from the desired marginal distributions. Posterior means of the various parameters were calculated based on retaining each 20th of the remaining cycles, i.e., 10,000 draws were used to estimate features of posterior distributions of interest.

\section{Model Comparison}

The 2 models were compared using the deviance information criterion (DIC) presented by Spiegelhalter et al. (2002). The DIC is defined as

$$
\mathrm{DIC}=\mathrm{D}+\mathrm{p}_{\mathrm{D}}
$$

where $\mathrm{D}$ is the posterior expectation of the Bayesian deviance and $\mathrm{p}_{\mathrm{D}}$ is a measure of the effective number of parameters in the model. Thus, the DIC considers both the fit of the model (as measured by D) and the number of penalties $\mathrm{p}_{\mathrm{D}}$ (proportional to number of parameters), which is necessary because models with an increased number of penalties have smaller deviance. Smaller values of DIC indicate a better fit of the model to the data.

Specifically,

$$
\mathrm{D}=\mathrm{E}_{\Theta y}[\mathrm{D}(\Theta)]
$$

where

$$
\mathrm{D}(\Theta)=-2 \log \mathrm{p}\left(\mathrm{y} \mid \Theta, \sigma_{\mathrm{e}}^{2}\right)
$$

We obtained D by calculating $\mathrm{D}(\Theta)$ every 2000 cycles (after the first 5000 cycles) and then averaging across all cycles. In addition, 
Table 1. Means and standard deviations (SD) of SCS in the entire sample and in healthy and infected udder halves.

\begin{tabular}{llll}
\hline Sample & Proportion & Mean & SD \\
\hline All $(\mathrm{n}=4518)$ & $\ldots$ & 4.59 & 2.87 \\
Healthy & 60.0 & 3.91 & 2.92 \\
Infected & 40.0 & 5.61 & 2.46 \\
\hline
\end{tabular}

${ }^{1}$ Healthy and infected status as determined by bacteriological culture.

$$
\mathrm{p}_{\mathrm{D}}=\mathrm{D}-\mathrm{D}(\bar{\Theta})
$$

where $\mathrm{D}(\bar{\Theta})$ is the Bayesian deviance (equation [8]) evaluated at the posterior mean of the parameters in $\Theta$. The posterior mean of $\Theta$ was calculated by averaging realizations of elements in $\Theta$ obtained every 500 cycles.

\section{Classification Ability}

As previously mentioned, bacterial culture data were available on a test-day basis for all udder-halves contributing data to the study. These records allowed for assessment of the ability of the FMM to correctly classify udder halves as healthy or infected on the date on which SCC was measured. Following Ødegard et al. (2003), 3 measures of classification ability were defined: 1) proportion of misclassification (PMC), 2) sensitivity, and 3) specificity.
The PMC was calculated for all observations. The vector $\mathbf{z}$ of classification variables was recalculated in each cycle of the sampler. To obtain the PMC, values of $z_{i}$ for each observation were summed across all cycles and divided by the number of cycles, yielding $\mathbf{m}$, a vector of the proportion of times each observation was assigned to the second component of the FMM. For each observation i from uninfected udder halves, $\mathrm{PMC}_{\mathrm{i}}=\mathrm{m}_{\mathrm{i}}$. For infected udder halves $\mathrm{i}, \mathrm{PMC}_{\mathrm{i}}=\left(1-\mathrm{m}_{\mathrm{i}}\right)$. The mean $\mathrm{PMC}$ was obtained by averaging the $\mathrm{PMC}_{\mathrm{i}}$. Sensitivity was the probability of correct classification of infected udder halves. Therefore, sensitivity was calculated by averaging $\mathrm{m}_{\mathrm{i}}$ for observations from the infected udder halves. Specificity was the probability of correct classification of healthy udder halves, and was 1 minus the average of $\mathrm{m}_{\mathrm{i}}$ for these observations. These classification statistics are available only for the FMM, so they were compared with values that would have been obtained by random classification, based on observed rates of infection. Finally, sensitivity of the FMM was calculated according to the type of bacteria causing the infection, inasmuch as different species of bacteria may induce different responses in terms of SCS.

\section{RESULTS AND DISCUSSION}

Table 1 has means and standard deviations of SCS for all goats, divided into healthy and infected groups.

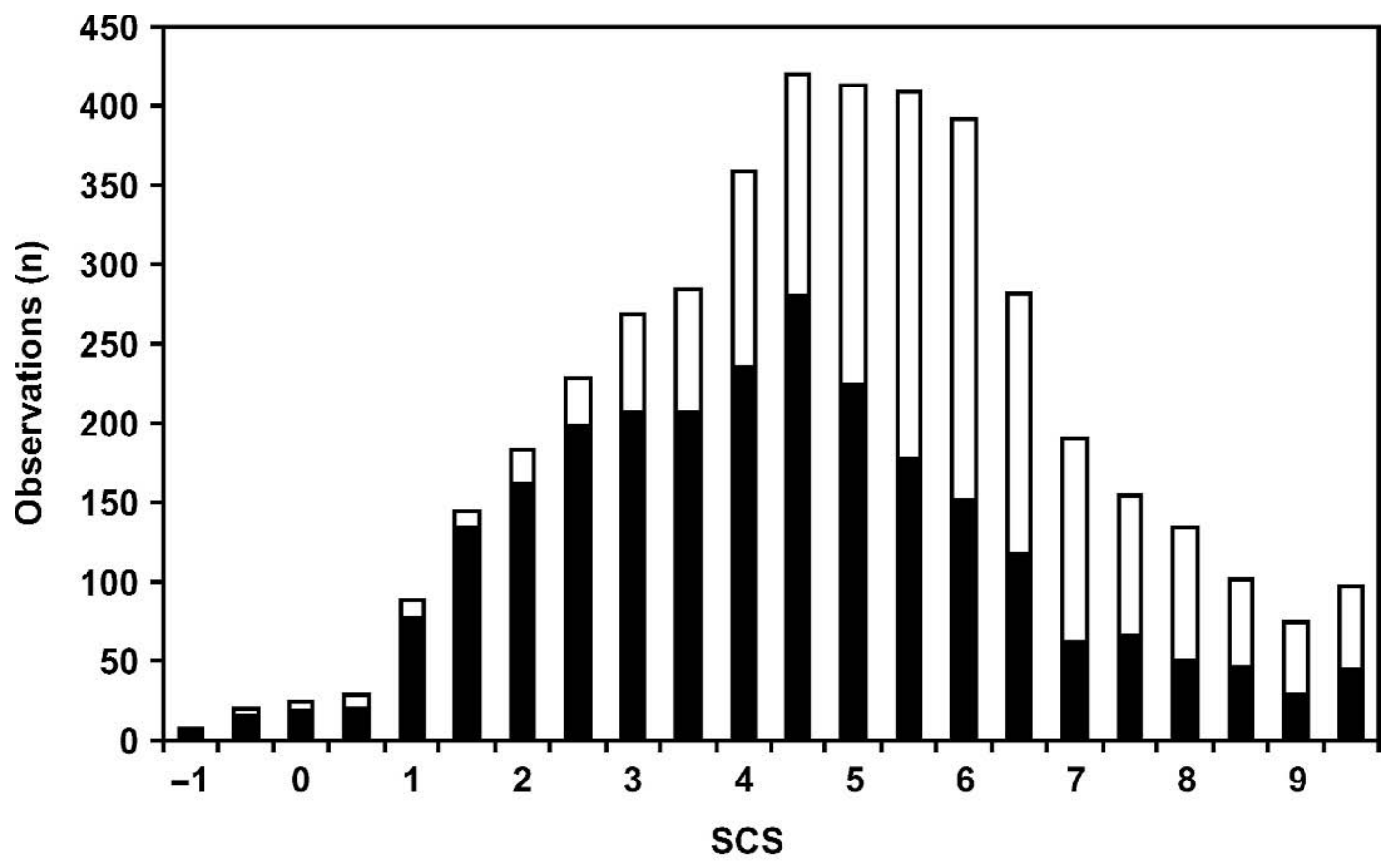

\section{Healthy $\square$ Infected}

Figure 1. Distribution of SCS for healthy and infected udder halves. 
Table 2. Posterior means and standard deviations (SD) for various parameters of interest from the standard and finite mixture models.

\begin{tabular}{|c|c|c|c|c|}
\hline \multirow[b]{3}{*}{ Parameter } & \multicolumn{4}{|c|}{ Model } \\
\hline & \multicolumn{2}{|c|}{ Standard } & \multicolumn{2}{|c|}{ Finite mixture } \\
\hline & Mean & SD & Mean & $\mathrm{SD}$ \\
\hline Proportion in "infected" group, \% & $\ldots$ & $\ldots$ & 43.7 & 5.8 \\
\hline Difference between groups, SCS & $\ldots$ & $\ldots$ & 2.83 & 0.60 \\
\hline Residual variance, SCS $^{2}$ & 3.02 & 0.07 & 1.28 & 0.19 \\
\hline Goat variance, $\mathrm{SCS}^{2}$ & 1.48 & 0.15 & 1.79 & 0.21 \\
\hline Udder half variance, SCS $^{2}$ & 0.18 & 0.04 & 0.18 & 0.05 \\
\hline
\end{tabular}

The distribution of SCS for healthy and infected udder halves is in Figure 1. The overall rate of infection in these herds was $40 \%$, which falls near the center of the range for infection rate reported by De Crémoux et al. (2001) based on a study of 155 dairy goat herds in France. The overwhelming majority $(80 \%)$ of the infections were CNS species, which are usually the most common source of subclinical mastitis in dairy goats (Bergonier et al., 2003). Environmental bacteria were responsible for $14 \%$ of the infections, and Staph. aureus caused 6\%. Mean SCS was 4.59, near the lower end of the range of SCS in goats reported in previous studies (e.g., McDougall and Voermans, 2002; Bergonier et al., 2003; Leitner et al., 2004). As expected, SCS was greater in the infected udder halves than in the uninfected udder halves. The difference in mean SCS between infected and uninfected halves was 1.70 SCS. For comparison, Lerondelle et al. (1992) reported a difference of 1.00 SCS, whereas Leitner et al. (2004) reported a difference of 2.07 SCS. Variability in SCS was larger among uninfected than infected udder halves, corroborating the observation of Haenlein (2002) that many factors other than bacterial infection influence SCC in dairy goats.

\section{Model Comparison}

Posterior means and standard deviations of parameters of interest from the 2 models are presented in Table 2 . The posterior mean and standard deviation of the mixing proportion were approximately 44 and 5.8\%, respectively. This agrees well with the observed rate of infection of $40 \%$ (Table 1). The posterior mean (SD) of the difference between means of the 2 components in the FMM was 2.83 (SD) SCS units. This is larger than the raw observed difference of 1.70 SCS, but the latter value entered at high density in a credibility region of size $95 \%$. This difference reflects the fact that the FMM accounts for variability due to other factors. Comparing estimates of variances from the 2 models, the most striking difference was for the residual variability. Residual variance was much less when esti- mated from the FMM than from the SLM (1.28 vs. 3.02 $\mathrm{SCS}^{2}$ ). This difference was clearly due to the fact that the FMM allowed for the definition of widely different means for the 2 components. In contrast, the variability due to the difference in these means was allocated into the residual variance for the SLM. The posterior mean of the variance of goat effects was also greater for the FMM, although the difference was not nearly as large as for the residual variance. Estimates of variances for udder halves were essentially the same in the 2 models. The combination of a decreased residual variance and an increased between-goat variance for the FMM might be construed, naively, as evidence that the FMM could produce a larger heritability. Genetic effects were not considered in our analysis, but repeatability is the upper limit for heritability. According to these results, the estimated repeatability $\left[\sigma_{\mathrm{g}}^{2} /\left(\sigma_{\mathrm{g}}^{2}+\sigma_{\mathrm{h}}^{2}+\sigma_{\mathrm{e}}^{2}\right)\right]$ was 0.31 for the SLM, and a similar calculation would yield 0.55 for the FMM. However, the correct expression for repeatability in our 2-component mixture model is

$$
\frac{\sigma_{g}^{2}}{\sigma_{g}^{2}+\sigma_{h}^{2}+\sigma_{e}^{2}+\left(1-P_{m}\right) \mu_{0}^{2}+P_{m} \mu_{1}^{2}-\left[\left(1-P_{m}\right) \mu_{0}+P_{m} \mu_{1}\right]^{2}}
$$

because the variance is mean-proportion dependent in a mixture model; $\mu_{0}$ and $\mu_{1}$ are the true means of SCS under the 2 distributions. On this basis, repeatability for the FMM was roughly 0.34 , slightly larger than for the linear mixed effects model.

In addition to having a smaller residual variance and a slightly larger repeatability, the FMM had a lower DIC than did the SLM. The DIC for the FMM was 21,679 vs. 55,834 for the SLM. As explained previously, a lower DIC is preferred in model comparison. The lower DIC of the FMM indicates that the improvement in fit to the data afforded by the FMM overwhelms the additional complexity introduced by the extra parameters.

\section{Classification Ability}

As mentioned previously, no classification is possible with the SLM, so the classification ability of the FMM 
Table 3. Sensitivity of the finite mixture model for different types of bacteria.

\begin{tabular}{ll}
\hline Species & Sensitivity \\
\hline Staphylococcus aureus & 59.1 \\
Coagulase-negative staphylococci & 48.0 \\
Streptococcus species & 42.9 \\
Environmental bacteria & 44.3 \\
\hline
\end{tabular}

was compared with random classification of observations based on observed rates of infection. Approximately $45 \%$ of observations were misclassified with the FMM. This compares to about 48\% PMC if observations had been assigned to components randomly, based on the observed proportion of healthy and infected udder halves. The proportion of $48 \%$ was obtained by assuming that $40 \%$ of the truly healthy udder halves would have been randomly assigned to the infected group and $60 \%$ of the observations from infected udder halves would have been randomly assigned to the healthy group.

The advantage of the FMM was in the classification of infected udder halves (sensitivity), which was $48 \%$ for the FMM, relative to $40 \%$ with random classification. In contrast, specificity of the FMM was about $60 \%$, equal to that for random classification of healthy udder halves. The rates of sensitivity and specificity observed in this study were much lower than the values obtained by Ødegard et al. (2003), and our level of PMC was much greater. However, a direct comparison between the 2 studies is not feasible. Ødegard et al. (2003) used simulated data, patterned after mastitis in dairy cattle where the proportion of healthy animals is around 70 to $85 \%$ and in which a greater proportion of mastitis infections show clinical symptoms.

The rates of sensitivity and specificity observed in this study are not high enough to justify the use of an FMM to diagnose individual cases of mastitis and identify animals that are candidates for treatment, at least in our goat population. Although sensitivity was greater than what could be achieved by random assignment, it was still below 50\%. In addition, the FMM analysis (as described here) can only be applied accurately after the fact, based on a large body of data, and thus too late to be of practical value for identification of infected animals. However, diagnosis of infections is not the sole purpose of such an analysis. The FMM represents a statistical improvement over the SLM, and thus may contribute to increased precision of genetic evaluations.

Rates of sensitivity varied according to the type of bacteria (Table 3). Sensitivity was greatest for udder halves infected with Staph. aureus (59.1\%). Previous researchers have reported strong associations between infections by Staph. aureus and elevated SCS, both at the cow (Schepers et al., 1997) and herd (Barkema et al., 1998) levels; Staph. aureus was also associated with the highest increase in SCS among the pathogens present in these herds (Moroni et al., accepted). Sensitivity for CNS infections was near the mean, $48 \%$, for all infections, as these bacteria are generally associated with a smaller increase in SCS than that produced by Staph. aureus. Sensitivity for environmental bacteria was nearly equal to that expected with random assignment to groups. Environmental bacterial infections are characterized by a spike in SCS followed by a relatively quick return to normal levels (de Haas et al., 2002).

Factors in addition to pathogen causing the infection also had effects on sensitivity. In general, factors that increased the difference in SCS between healthy and infected animals increased the sensitivity of the FMM. For example, Figure 2 shows the trend of sensitivity and SCS for healthy and infected udder halves with DIM. Sensitivity was much greater in early lactation (approximately 60 to $70 \%$ ) than in late lactation (around 40\%). During this period (up to $90 \mathrm{~d}$ ), mean SCS among infected individuals was 2 to 3 times greater than was mean SCS of healthy individuals. The mean SCS tended to increase with increasing DIM in both groups, but for uninfected udder halves the increase was at a greater rate. Hence, at the end of lactation, the mean of uninfected udder halves (6.53) was only slightly less than that for those with infections (7.05). As a result, sensitivity during this period was rather low, at $36 \%$.

From a purely statistical point of view, the FMM was superior to the SLM for analysis of test-day SCS. Residual variance from the FMM was $60 \%$ lower than from the SLM. Repeatability was slightly larger, suggesting that the FMM would yield larger estimates of heritability and, perhaps, more precision in genetic evaluation of SCS. These results suggest that more research on the use of an FMM for the analysis of SCS data is merited.

This empirical study leaves questions that must be answered before any practical application. The next logical step would be to increase the complexity of the FMM used in this analysis and to apply it to other data. A first extension would be to include genetic effects. Ødegard et al. (2003) applied such a model to simulated data, but no applications to real data have been reported yet. Another useful generalization would be to allow for heterogeneous variances. Ødegard et al. (2003) also applied this extension, but to simulated data only. Experimentation with heterogeneous variances for genetic effects and an imperfect genetic correlation between the alternative states could be particularly valuable for practical applications (Gianola et al., 2004). 


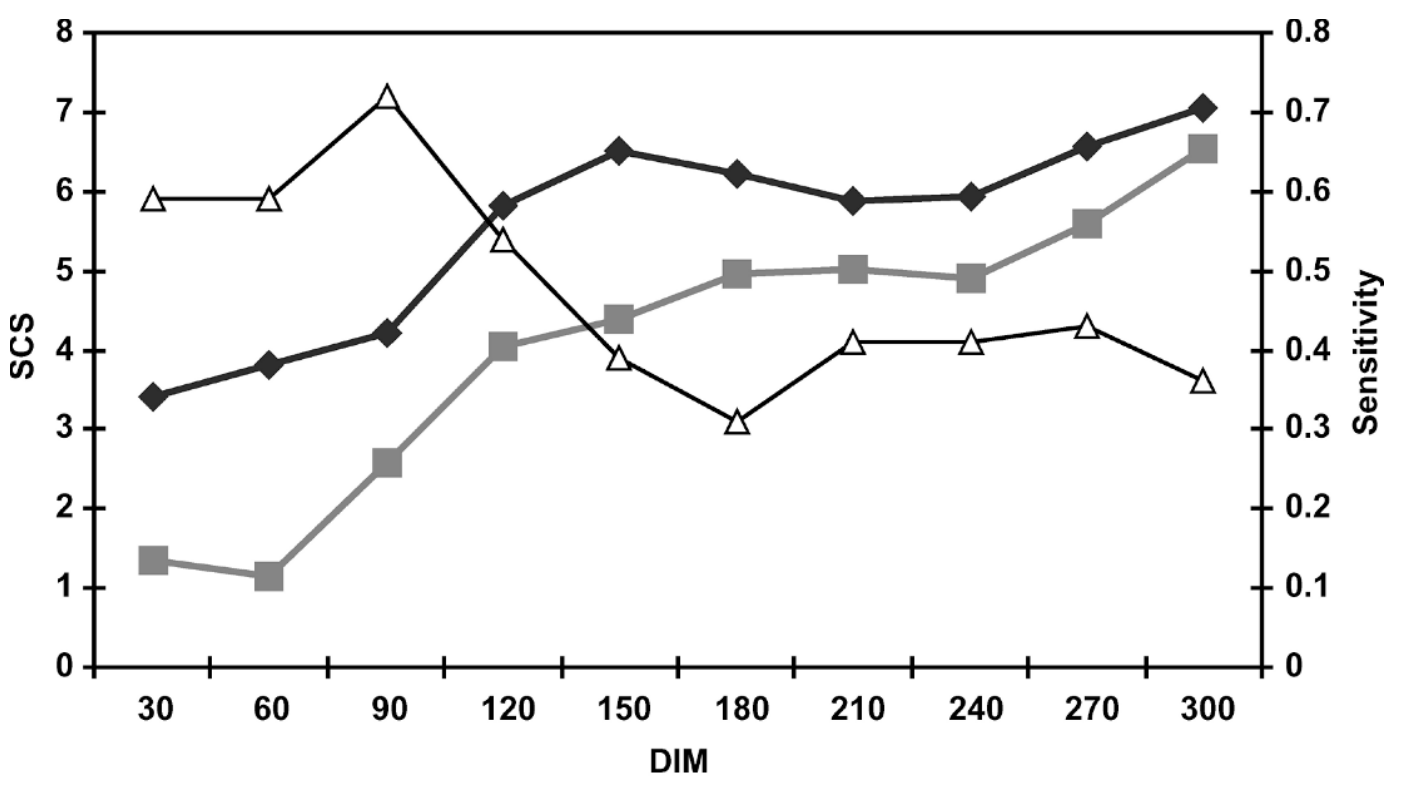

- Healthy SCS $\leadsto$ Infected SCS $\leadsto-$ Sensitivity

Figure 2. Trends in SCS for healthy and infected udder halves and sensitivity of the finite mixture model across lactation.

Somatic cell scores may be different traits genetically for healthy and infected animals (Detilleux and Leroy, 2000), and an FMM with heterogeneous variances may be the only way to account for these different traits if mastitis incidence is not recorded.

Following development of a more complex FMM for SCS, the next critical step would be evaluation of the biological meaning of results. Our study indicates that the FMM is superior to the SLM from a statistical point of view, and that it may result in increased heritability and more precise genetic evaluations. From a practical standpoint, the advantages of applying the FMM may be greater for dairy cattle than for dairy goats. Although SCS tends to be elevated among goats with udder infections (e.g., Leitner et al., 2004; Moroni et al., accepted), the effect of infection on SCS tends to be greater in dairy cattle. For a number of reasons, including higher natural levels of SCC in goat's milk than in cow's milk (Droke et al., 1993), poor specificity of cell counting machinery (Poutrel and Lerondelle, 1983), and strong effects of other factors such as estrus (Aleandri et al., 1996) on SCC in goats, SCC is considered a less reliable indicator of mastitis in goats than in cattle. In fact, routine genetic evaluation of goats for SCC is essentially nonexistent.

Regardless of the species to which an FMM analysis of SCS is applied, certain practical issues must be considered. The EBV for SCS derived from an FMM may not have the same genetic meaning as EBV from the SLM. Males with high (unfavorable) EBV for SCS gen- erally have high EBV not simply because their daughters have relatively greater SCS than herdmates in both the infected and uninfected states, but also because they tend to have more daughters in the infected state. As suggested by Ødegard et al. (2003), the FMM could be extended to allow for genetic influences on the mixing proportion, $\mathrm{P}_{\mathrm{m}}$, which could thus capture differences in sires for the probability that their daughters' records will be assigned to the healthy or infected group. Such an extension was recently presented by Ødegard et al. (accepted). Once an appropriate FMM for SCS is arrived at, indices would need to be developed, so that genetic response for mastitis resistance can be optimized.

\section{ACKNOWLEDGMENTS}

This research was partly funded by the Wisconsin Agriculture Experiment Station and by grants NRICGP/USDA 2003-35205-12833 and NSF DEB0089742. The authors would like to thank the producers that participated in the study.

\section{REFERENCES}

Aleandri, M., A. Fagiolo, P. Calderini, R. Colafrancesco, G. Giangolini, R. Rosati, and F. Demichelis. 1996. Studies conducted on somatic cells count of goat milk. Pages 65-70 in Somatic Cells and Milk of Small Ruminants. R. Rubino, ed. EAAP Publication No. 77. Wageningen Pers, Wageningen, The Netherlands.

Ali, A. K. A., and G. E. Shook. 1980. An optimum transformation for somatic cell concentration in milk. J. Dairy Sci. 63:487-490. 
Barkema, H. W., Y. H. Schukken, T. J. G. M. Lam, M. L. Beiboer, H. Wilmink, G. Benedictus, and A. Brand. 1998. Incidence of clinical mastitis in dairy herds grouped in three categories by bulk milk somatic cell counts. J. Dairy Sci. 81:411-419.

Bergonier, D., R. De Crémoux, R. Rupp, G. Lagriffoul, and X. Berthelot. 2003. Mastitis in small dairy ruminants. Vet. Res. 34:689-716.

De Crémoux, R., V. Heuchel, and F. Berny. 2001. Description et interprétation des comptages de cellules somatiques des laits de troupeaux en élevage caprin. Proceedings of Rencontres Recherches Ruminants 8:163. INRA, Paris, France.

de Haas, Y., H. W. Barkema, and R. F. Veerkamp. 2002. The effect of pathogen-specific clinical mastitis on the lactation curve for somatic cell count. J. Dairy Sci. 85:1314-1323.

Detilleux, J., and P. L. Leroy. 2000. Application of a mixed normal mixture model for the estimation of mastitis-related parameters. J. Dairy Sci. 83:2341-2349.

Droke, E. A., M. J. Paape, and A. L. Di Carlo. 1993. Prevalence of high somatic cell counts in bulk tank goat milk. J. Dairy Sci. 76:1035-1039.

Gianola, D., J. Odegard, B. Heringstad, G. Klemetsdal, D. Sorensen, P. Madsen, J. Jensen, and J. Detilleux. 2004. Mixture model for inferring susceptibility to mastitis in dairy cattle: A procedure for likelihood-based inference. Genet. Sel. Evol. 36:3-27.

Haenlein, G. W. 2002. Relationship of somatic cell counts in goat milk to mastitis and productivity. Small Rumin. Res. 45:163-178.

Interbull. 1996. Sire evaluation procedures for non-dairy-production and growth and beef production traits practised in various countries. Bull. No. 13. Int. Committee Anim. Recording, Uppsala, Sweden.

Leitner, G., U. Merin, and N. Silanikove. 2004. Changes in milk composition as affected by subclinical mastitis in goats. J. Dairy Sci. 87:1719-1726.
Lerondelle, C., Y. Richard, and J. Issartia. 1992. Factors affecting somatic cell counts in goat milk. Small Rumin. Res. 8:129-139.

Liu, Y., G. B. Jansen, and C. Y. Lin. 2004. Quantitative trait loci mapping for dairy cattle production traits using a maximum likelihood method. J. Dairy Sci. 87:491-500.

McDougall, S., and M. Voermans. 2002. Influence of estrus on somatic cell count in dairy goats. J. Dairy Sci. 85:378-383.

McLachlan, G., and D. Peel. 2000. Finite mixture models. 1st ed. John Wiley and Sons, New York, NY.

Moroni, P., G. Pisoni, G. Ruffo, and P. J. Boettcher. Influence of intramammary infection and parity on somatic cell counts in Italian dairy goats. Prev. Vet. Med. (accepted)

Ødegard, J., J. Jensen, P. Madsen, D. Gianola, G. Klemetsdal, and B. Heringstad. 2003. Detection of mastitis in dairy cattle by use of mixture models for repeated somatic cell scores: A Bayesian approach via Gibbs sampling. J. Dairy Sci. 86:3694-3703.

Ødegard, J., P. Madsen, D. Gianola, G. Klemetsdal, J. Jensen, B. Heringstad, and I. R. Korsgaard. A Bayesian threshold-normal mixture model for analysis of a continuous mastitis-related trait. J. Dairy Sci. (accepted)

Poutrel, B., and C. Lerondelle. 1983. Cell content of goat milk: CMT, Coulter Counter and Fossomatic for predicting half infection. J. Dairy Sci. 66:2575-2579.

Schepers, A. J., T. J. G. M. Lam, Y. H. Schukken, J. B. M. Wilmink, and W. J. A. Hanekamp. 1997. Estimation of variance components for somatic cell counts to determine thresholds for uninfected quarters. J. Dairy Sci. 80:1833-1840.

Spiegelhalter, D. J., N. G. Best, B. P. Carlin, and A. van der Linde. 2002. Bayesian measures of model complexity and fit. J. R. Stat. Soc. Ser. B Stat. Methodol. 64:583-639.

Wilmink, J. B. M. 1987. Adjustment of test-day milk, fat and protein yield for age, season and stage of lactation. Livest. Prod. Sci. 16:335-348. 